Research Article

\title{
Facial Gesture Measurement Using Optical Muscle Sensing System
}

\author{
Kriengsak Yothapakdee ${ }^{1}$, Preecha P. Yupapin ${ }^{3}$, Kreangsak Tamee ${ }^{1,2}$ \\ ${ }^{1}$ Department of Computer Science and Information Technology, Faculty of Science, Naresuan University, Phitsanulok 65000, \\ Thailand \\ ${ }^{2}$ Research Center for Academic Excellence in Nonlinear Analysis and Optimization, Faculty of Science, Naresuan University, \\ Phitsanulok 65000, Thailand \\ ${ }^{3}$ Interdisciplinary Research Center, Faculty of Science and Technology, KasemBundit University, Bangkok 10250, Thailand
}

Corresponding author: E-mail: kreangsakt@nu.ac.th

Received: Oct. I, 2015; Accepted: Dec. 30, 2015; Published: Jan. 22, 2016

Citation: Kriengsak Yothapakdee, Preecha P. Yupapin and Kreangsak Tamee. Facial Gesture Measurement Using Optical Muscle Sensing System. Nano Biomed. Eng. 2015, 7(4), 169-179.

DOI: 10.5I01/nbe.v7i4.pI69-179.

\begin{abstract}
A PANDA ring resonator circuit has been applied to the measurement of muscle actions, measuring signals created by facial muscle contractions. A system, which is called the Optical Muscle Sensing System, was developed which uses sensors to measure the mechanism of facial muscle contractions and the strength of contraction and degrees of perturbation of the facial muscles that are used directly for each facial gesture. The signal data was obtained from the simulation of the facial gestures and this data was applied in the classification of the facial gesture signals of each particular gesture. Facial gestures include blinking, smiling, grimacing and various other contortions of the face which may imply emotions and are part of normal human communication. Understanding of these mechanisms will be useful and applicable to facial rehabilitation services.
\end{abstract}

Keywords: Facial gesture measurement; Optical muscle sensing system; Optical sensor Facial gesture signals; PANDA ring resonator; Facial gesture recognition

\section{Introduction}

Human gestures or body language is recognized as playing an important role in human communication which complements verbal communication. Gesturing and body posture, arm and hand gestures, face movement and pose, facial gestures and eye movement and gaze are all important communication patterns. Such body language provides a powerful source of communicative information in a human gesture recognition system [1]. The objective of developing and improving these technologies is to provide various powerful applications to assist the disabled and elderly in normal daily activities and in rehabilitation and support services. Applications that have been developed have been for use in Human-Computer Interaction (HCI) or Human-Machine Interaction (HMI), and are generally known as Sensory Substitute Systems.

Scientific researchers in the field of rehabilitation engineering have attempted to develop various devices for helping patients, the disabled and the elderly [2-4] and there has been considerable success in achieving improvements to the quality of life for these people 
by the application and use of these various devices. However, these devices have usually been based on the physical movement of the body, particularly the head, arms, fingers, legs and feet, and these devices often have use and usefulness to only certain user groups. For example, most of these devices are not useful or appropriate to people with paralysis of part or all of the body.

Nonetheless, certain levels of muscle weakness and physical movement limitations have been improved and physical capabilities developed through the use of devices that the measured and analyzed electrical signals acquired from the body, called Bio-signals $[5,6]$. These signals originate at the molecular level, cell level or at the systemic or organ level [7]. Examples of well-known and detectable biosignals include electromyograms (EMG) which emanate from the electrical activity from the muscles. Electro-oculograms (EOG) are signals detected from eye movements as are electroretinograms (ERG) from the eye. Electrical signals from heart muscle activity; the electrocardiogram (ECG) as well as the electroneurogram (ENG); field potentials from local regions in the brain are all examples of detectable signals from muscles and organs in the body. These bio-signals have been identified and are detectable and are widely used in various control applications such as controlling a motorized wheelchair by EOG signals $[8,9]$, a glove which is a force-audio sensory substitution system for diabetic patients [10], and a visual substitution system for blind people [11, 12]. Particularly, Facial Gesture Recognition (FGR) offers a challenges and is certainly of interest. Such as system uses human gestures in application of robotic control and computer vision, generally known as HumanRobot Interaction (HRI) [13-15]. These systems have been investigated with a focus on the advantages of using human gesture recognition in many application areas [16-19]. Unfortunately, these devices still problems with over-heating after continuous use for a long time with continuous energy consumption. Heating within the circuitry of the device is also usual, as a chemical reaction, oxidization and corrosion. Therefore, optical devices are the only alternative.

Optical devices can be used to replace mechanical devices or parts of such devices, or increase the efficiency and effectiveness of those devices and applications, now, and certainly in the future. The advantages of optical devices include being immune to radio frequency interference (RFI) and electromagnetic interference (EMI), being very small, passive and with low power consumption, having excellent performance with high sensitivity and wide bandwidth and being non-contaminating of their environment. Finally, they are not subject to corrosion [20]. Optical sensors based on a ring resonator have been used in robotic control systems, security applications and in monitoring and detection applications in industry, such as production line inspections and quality control.

One advanced application is their use in nanoscale sensors, which is a specific model of PANDA ring resonator type reported by P.P. Yupapin and et al [21]. They have been developed for and applied to a wide variety of applications; distributed sensors [22], molecular sensors [23], gas sensors [24] and force sensing application [25]. Recently, Yupapin and Sarapat have shown the advanced optical sensing system that uses the whispering gallery modes (WGMs) of light within a micro-scale modified add-drop filter circuit for various sensing applications [26]. In our previous research [27-28] we developed a conceptual framework and device model of a muscle measurement system using optical devices which measure muscle contractions. As well, the results from our previous research can be used for pattern recognition. The PANDA ring resonator circuit still has obstacles associated with limitations in materials, sophisticated production technology and relatively high production costs. These factors have been obstacles to the fabrication of PANDA ring circuits which are currently not being produced.

Thus, we have proposed an approach which uses simulation of the processes of facial gesture measurement using what we term the Optical Muscle Sensing System. In this system, in order to have consistent facial gesture signal data which is as close as possible to measurements from a real situation, we generate a signal based on the theory of muscle contraction and optical sensing mechanisms. The simulations were conducted by using the MATLAB and Opti-wave software programs. Although our performance is a simulated work, we are confident that the same device parameters and methodologies can be applied to real devices, which will enable them to be fabricated and implemented in the near future. This is an important advance towards making these optical devices useful for nano-scale sensing systems, human computer interaction, human-robot interaction and in disability applications. 


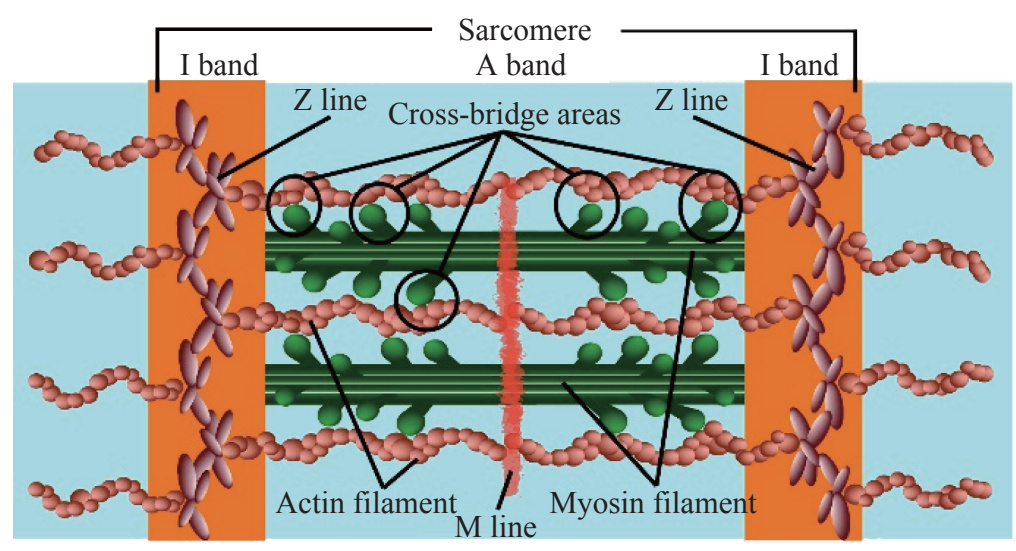

Fig. 1 A cross-bridge areas within sarcomere, and the contractile actin and myosin filaments.

\section{Muscular Contraction and Optical Sensing Mechanism}

Almost all of the procedures or mechanisms that affect the process of movement of the organs in the mammalian body will be based on the muscular contraction mechanisms. Typically, the muscles can be categorized into three types; skeletal, cardiac, and smooth. The skeletal muscles are voluntary muscles that allow us to control the gestures and the movements of our body. The skeletal muscles are composed of bundles of muscle fibers or cells, each of which are composed of sub-units called myofibrils, which are composed of many myofibrils, organized into contractile units of skeletal muscles called sarcomeres. These sarcomeres are the smallest functional unit of the muscle fiber and are responsible for muscle contraction. In the past, the main mechanism for muscle contraction has been described as principles of interaction between the two sets of protein myofibrils within the sarcomere known as the sliding filament theory [29], which is pulling the Z-lines (bundles of fibrous cells responsible for separate and link sarcomeres within a skeletal muscle) towards the center of the sarcomere. Such reactions are the cause of the shortened muscle fibers as shown in Fig. 1.

The cross-bridge theory [30-32] is now recognized as the paradigm of a suitable approach to describing and simulating the mechanism of muscle contraction. According to this theory, the muscle contraction occurs through the interaction of myofibrils which is the main component inside the sarcomere. The simple working model of the cross-bridge cycle could explain how the myosin filament head moves in order to attach the actin filament, thereby forming a sort of cross bridge between the two filaments as shown in Fig. 1.
The force of muscle contraction can be detected and measured based on the number of myosin and actin cross bridges that are attached. This in turn is affected by four factors [33]: (1) the number of muscle fibers stimulated, (2) the relative size of the fibers, (3) the frequency of stimulation and (4) the degree of muscle stretch, which can be divided into three periods: (1)
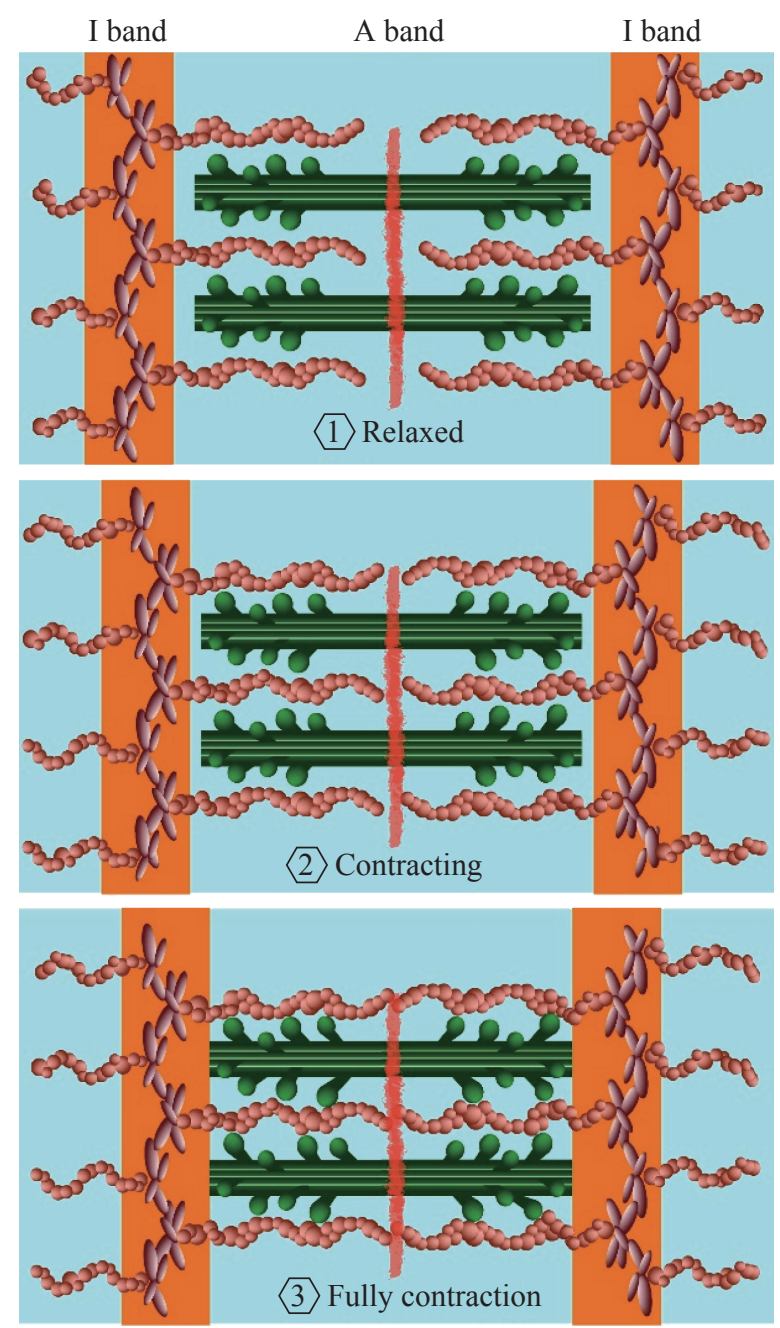

Fig. 2 The individual degree of muscle contraction. 
sarcomeres greatly shortened, (2) sarcomeres at resting length, and (3) sarcomeres excessively stretched. Here, we call these the relaxed state, the contracted state, and the full contracted state respectively, as show in Fig. 2.

Fig. 3, from [34], shows the dependence of force on sarcomere length. The shortening of the sarcomere from (A) to (B) $3.65 \mu \mathrm{m}$ to $2.25 \mu \mathrm{m}$ results from the relaxation of the muscle, (B) to (D) $2.24 \mu \mathrm{m}$ to 1.67 $\mu \mathrm{m}$ is the contraction of muscles, and (D) to (E) 1.66 $\mu \mathrm{m}$ to $1.27 \mu \mathrm{m}$ is full contracted of muscles. So, the

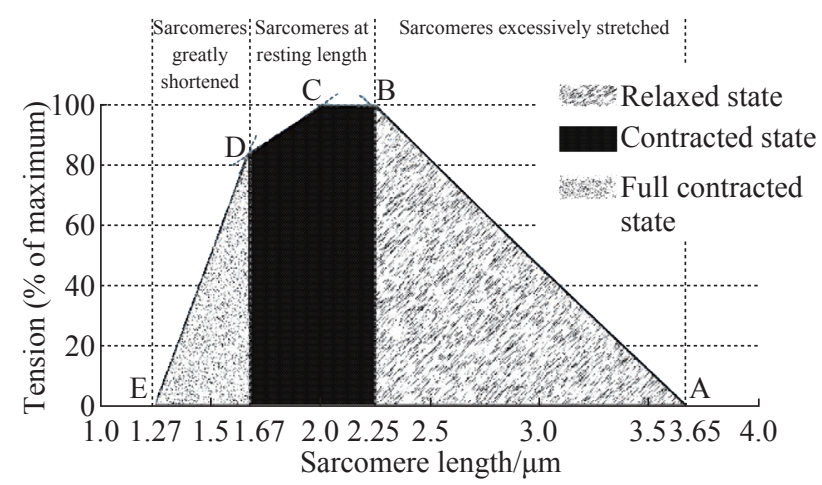

Fig. 3 The relationship between the shortening of the sarcomere and the sarcomere length [34]. relationship between the sarcomere length and the degree of muscle contraction can be described by a shortening of the muscle.

The Optical Muscle Sensing System has been devised and modified on the basis of a PANDA ring resonator circuit. The schematic diagram of the system, which consists of three micro-ring resonators, is shown in Fig. 4(1). The first ring is arranged as a reference unit $\left(R_{\mathrm{L}}\right)$. The second ring $\mathrm{RR}$ is the sensing unit, and the third ring is used to form interference signals between the signals from the first ring and the second ring. The waveguide material and suitable parameter configurations are well described in [21-25].

The explanation of the method performed within the Optical Muscle Sensing System can be categorized into two cases, the non-perturbed situation and the perturbed situation. In the first case, the part of the sensing unit $\left(R_{R}\right)$ non-perturbed by the muscle contraction, can be simulated by introducing Gaussian beams into the input port of the PANDA ring resonator circuit. The output signal from both the reference unit $\left(E_{L}\right)$ and the sensing unit $\left(E_{R}\right)$ can then be detected at the drop port. The relationship between the peaks of

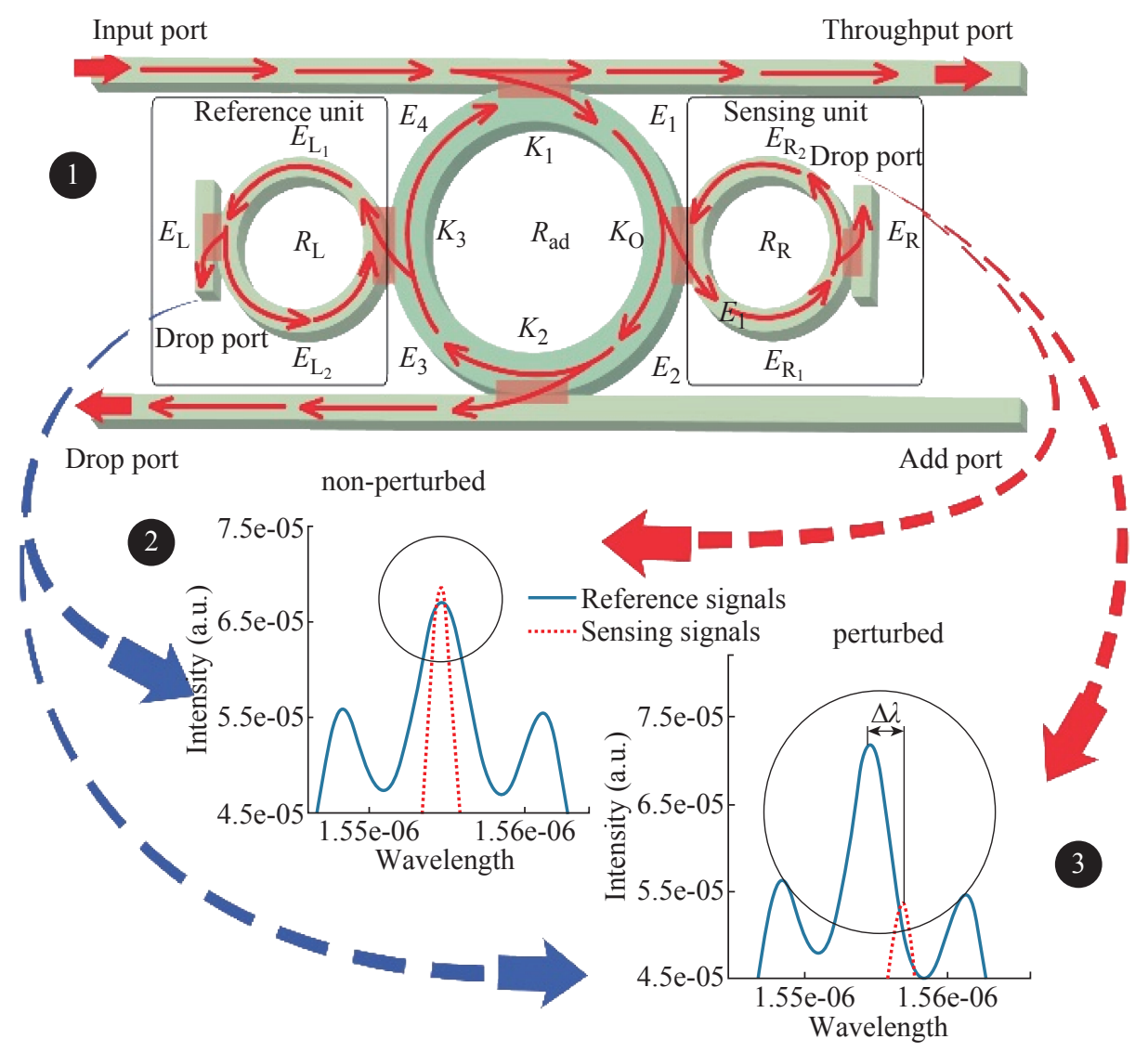

Fig. 4 Schematic diagram of the optical muscle sensing system, where (1) a PANDA ring resonator, the comparison of signals between the reference and the sensing signals in non-perturbed situation (2), and in perturbed situation (3). 
the reference signal (blue line) and the sensing signal (red line) are plotted, as shown in Fig. 4(2), which demonstrates that the peaks of the wavelengths of both signals have not shifted. In the second case, the part of the sensing unit $\left(R_{R}\right)$ that has been perturbed by the muscle contraction, can also be simulated by introducing Gaussian beams into the input port of the PANDA ring resonator circuit. A comparison done at the drop port of both units, clearly indicates that the peak of the wavelength within the reference unit and the sensing unit has shifted, as shown in Fig. 4(3).

The relationships between the change in wavelength and the perturbation of the muscle contraction can be explained through the principle of the change within the optical path length. Because the muscle contraction mechanism to perturb the sensing unit $\left(R_{R}\right)$ is the cause of the change affected within the optical path length $(L)$, this directly affects the wavelength shift $(\Delta \lambda)$ by Eq. (1).

$\frac{\Delta \lambda_{m}}{\lambda_{m}}=\frac{\Delta n}{n}+\frac{\Delta L}{L}$

here, $m$ is an integer, $n$ is the refractive index of the guiding material, and $L$ is the optical path length of the ring resonator.

For the relationship between the force and the change in the sensing unit $R_{R}$, the length is described by

$$
F=\left(\frac{Y_{0} A_{0}}{L_{0}}\right) \bullet \Delta L
$$

where $F$ is the applied force, $Y_{0}$ is the Young's modulus and $A_{0}$ is the initial cross-section area. $L_{0}$ is the initial length and $(\Delta L)$ is the change in length. Thus, the sensor principles can apply to muscle measurements. The linear relationship between the applied force and the wavelength shift $(\Delta \lambda)$ is formed. This linearity indicates that this relationship is appropriate for use in the Optical Muscle Sensing System as shown in Fig. 5.

\section{Facial Gesture Signal Generation}

Our system was based on the assumption that the Optical Muscle Sensing System can sense and measure facial gestures based on the contraction of the facial muscle used in the gesture. In this research, we divided the facial muscles into 8 facial gestures which are formed by the facial muscles. We observed the reaction and response to the facial muscles. These relationships between the facial gesture and the particular effective muscle or muscles are well known. This relationship is

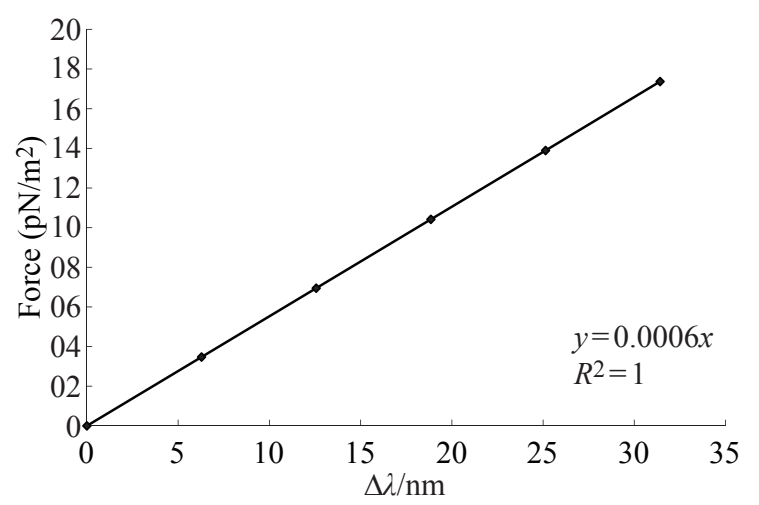

Fig. 5 Graph of the linear relationship between force and the wavelength shift $(\Delta \lambda)$.

Table 1 The facial gestures and effective muscles [33-35]

\begin{tabular}{|c|c|}
\hline Elementary facial gestures & Facial muscle involved \\
\hline Closing left eye & $\begin{array}{c}\text { Orbicularis oculi (left) } \\
\text { Corrugator supercilii (left) } \\
\text { Frontalis (left) } \\
\text { Procerus }\end{array}$ \\
\hline Closing right eye & $\begin{array}{c}\text { Orbicularis oculi (right) } \\
\text { Corrugator supercilii (right) } \\
\text { Frontalis (right) } \\
\text { Procerus }\end{array}$ \\
\hline Closing both eyes & $\begin{array}{l}\text { Orbicularis oculi } \\
\text { Corrugator supercilii } \\
\text { Frontalis } \\
\text { Procerus }\end{array}$ \\
\hline Pull up the eyebrows & $\begin{array}{c}\text { Levator palpebrae superioris } \\
\text { Corrugator supercilii } \\
\text { Frontalis } \\
\text { Procerus }\end{array}$ \\
\hline Smiling & $\begin{array}{c}\text { Zygomaticus major } \\
\text { Zygomaticus minor } \\
\text { Risorius } \\
\text { Buccinator }\end{array}$ \\
\hline Smiling with left side & $\begin{array}{c}\text { Zygomaticus major (left) } \\
\text { Zygomaticus minor (left) } \\
\text { Risorius (left) } \\
\text { Buccinator (left) }\end{array}$ \\
\hline Smiling with right side & $\begin{array}{c}\text { Zygomaticus major (right) } \\
\text { Zygomaticus minor (right) } \\
\text { Risorius (right) } \\
\text { Buccinator (right) }\end{array}$ \\
\hline Clenching molar teeth & $\begin{array}{c}\text { Zygomaticus major } \\
\text { Zygomaticus minor } \\
\text { Risorius } \\
\text { Buccinator }\end{array}$ \\
\hline
\end{tabular}

shown in Table 1.

We identified eleven locations for placing the sensing probes which provide feedback to the system. The data collected using these probes are used by the Optical Muscle Sensing System in order to detect and measure the individual facial gesture signals and to confirm that they are appropriately similar to the real situation. As illustrated in Fig. 6, these eleven locations cover all of the main facial muscle components used in the mechanisms of facial expressions and facial 


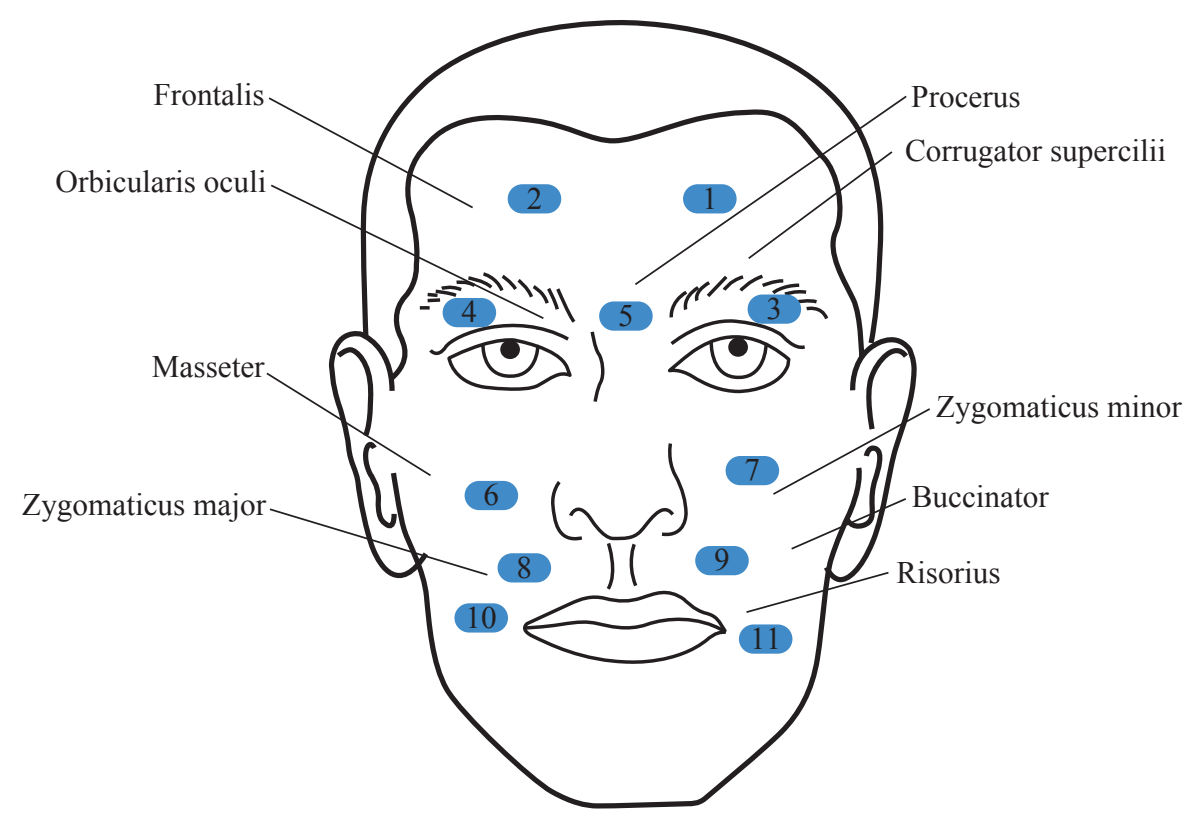

Fig. 6 Sites of 9 implicated facial muscles and 11 the feature probes.

gestures. We did confirm that the attributes of these eleven locations, and the data returned by the probes, are sufficient for classification of all facial gestures. The location of the probes included the frontal is (occipitofrontalis) muscle, for probes $\mathrm{P} 1$ and $\mathrm{P} 2$, the orbicularis oculi and corrugator supercilii muscles for probes $\mathrm{P} 3$ and $\mathrm{P} 4$, the procerus muscle for probe $\mathrm{P} 5$, the zygomaticus minor and masseter muscles for probes $\mathrm{P} 6$ and $\mathrm{P} 7$, the zygomaticus major muscle for probes $\mathrm{P} 8$ and $\mathrm{P} 9$, and the risorius muscle for probes F10 and P11.

The next step in the development of the Optical Muscle Sensing System, was to define the method of calculating the length of sarcomeres in each degree of muscle contraction. The degrees of shortening of the sarcomeres within the muscle contraction mechanism depends on the transformation of the sarcomeres which is based on the nature of the cross-bridge theory. Similarly, the length-tension of the muscle for each degree of muscle contraction is based on the shortening of the sarcomere, as shown in Fig. 3. The length of the sarcomeres is therefore given by functions (3), (4) and (5),

$$
\begin{aligned}
& \Delta S_{\text {relaxed }}=\Delta S_{A \rightarrow B}=3.65-2.25=1.40 \\
& \Delta S_{\text {contracting }}=\Delta S_{B \rightarrow D}=2.25-1.67=0.58 \\
& \Delta S_{\text {full-contracted }}=\Delta S_{D \rightarrow E}=1.67-1.27=0.40
\end{aligned}
$$

where $\Delta S_{\text {relaxed }}, \Delta S_{\text {contracting, }}$, and $\Delta S_{\text {full-contracted }}$ are the lengths of sarcomeres in relaxed status, initiated or contracting status, and fully contracted status. The percentage of the length of the sarcomeres can be obtained by multiplying the length of the sarcomere by 100 and divide by $\Delta S_{\text {relaxed }}+\Delta S_{\text {contracting }}+\Delta S_{\text {full-contracted }}$ as shown in functions (6), (7) and (8),

$$
\begin{aligned}
& \% \Delta S_{\text {relaxed }}=\frac{\Delta S_{\text {relaxed }} \times 100}{\Delta S_{\text {relaxed }}+\Delta S_{\text {contracting }}+\Delta S_{\text {full-contracted }}} \\
& \% \Delta S_{\text {contracting }}=\frac{\Delta S_{\text {contracting }} \times 100}{\Delta S_{\text {relaxed }}+\Delta S_{\text {contracting }}+\Delta S_{\text {full-contracted }}} \\
& \% \Delta S_{\text {full-contracted }}=\frac{\Delta S_{\text {full-contracted }} \times 100}{\Delta S_{\text {relaxed }}+\Delta S_{\text {contracting }}+\Delta S_{\text {full-contracted }}}
\end{aligned}
$$

For example, $\% \Delta S_{\text {relaxed }}=(1.40 \times 100) /(1.40+0.58$ $+0.40)=58.82 \%, \% \Delta S_{\text {contracting }}$ and $\% \Delta S_{\text {full-contracted }}$ are $24.37 \%$ and $16.81 \%$, as shown in Column 2 of Table 2. We have determined the degrees of sensor impacts based on the degrees of the sliding filament mechanism, which is consistent with the degrees of facial muscle contraction. In the previous paragraph, the calculated methods of the lengths of sarcomeres and the percentage of the lengths of the sarcomeres were presented.

Next, the degrees of the perturbed optical muscle sensing system based on the degrees of the sliding filament mechanism, which is consistent with the degrees of the facial muscle contraction are explained and defined. In order to the degrees of the perturbation have an effect on the sensing system was similar and consistent with the actual degrees of the shortening of sarcomere lengths. Hence, we have divided the degrees of sensor perturbations into three levels include the "Affecter", which was used for the contraction of 
Table 2 The degrees of the sliding filament compared to the degrees of sensor perturbations

\begin{tabular}{ccc}
\hline Degrees of the sliding filament mechanism & The percentage of sarcomeres length & Degrees of sensor perturbations \\
\hline Relaxed & 58.82 & Affecter \\
Contracting & 24.37 & Minor \\
Fully contracted & 16.81 & Major \\
\hline
\end{tabular}

Table 3 The degrees of affect various probe

\begin{tabular}{|c|c|c|c|c|c|c|c|c|c|c|c|}
\hline \multirow{2}{*}{ Gesture Name } & \multicolumn{11}{|c|}{ Perturbation level of probes } \\
\hline & P1 & $\mathrm{P} 2$ & P3 & P4 & P5 & P6 & P7 & P8 & P9 & P10 & P11 \\
\hline Closing both eyes & Minor & Minor & Major & Major & Major & Minor & Minor & Minor & Minor & Affecter & Affecter \\
\hline Closing left eye & Major & Minor & Major & Affecter & Major & Affecter & Major & Minor & Major & Affecter & Minor \\
\hline Closing right eye & Minor & Major & Affecter & Major & Major & Major & Affecter & Major & Minor & Minor & Affecter \\
\hline Pull up the eyebrows & Major & Major & Major & Major & Minor & Minor & Minor & Minor & Minor & Affecter & Affecter \\
\hline Smiling & Affecter & Affecter & Minor & Minor & Affecter & Major & Major & Major & Major & Minor & Minor \\
\hline Smiling with left side & Minor & Minor & Major & Affecter & Minor & Affecter & Major & Minor & Major & Affecter & Major \\
\hline Smiling with right side & Minor & Minor & Affecter & Major & Minor & Major & Affecter & Major & Minor & Major & Affecter \\
\hline $\begin{array}{l}\text { Clenching molar teeth } \\
\text { (rage) }\end{array}$ & Minor & Minor & Minor & Minor & Affecter & Major & Major & Major & Major & Minor & Minor \\
\hline
\end{tabular}

muscles in a relaxed state, the "Minor" which was used for the contraction of muscles in contracting state, and the "Major" which was used for the contraction of muscles in a fully contracted state. This is shown in Column 3 of Table 2. In the same way, the differences in the perturbed levels during the contraction of each the facial muscle is being formed, which is be based on the facial gestures are shown in Table 3. As identified in Fig. 6, we can assume that the facial gesture "Pull up the eyebrows", for example, is controlled by particular muscles and the degrees of stimulation force can be sensed by the various probes in the affected areas. Overall, the affect (perturbed level) of each particular facial gesture on each probe is shown in Table 3. Therefore, any specific facial gesture will affect the particular probes which measure the stimulation force asserted on the facial muscle by the facial gesture.

\section{Simulation Results}

To form the simulation of the facial gesture measurement by using the optical muscle sensing system, the parameters of the PANDA ring resonator were fixed to be the Gaussian beams wavelength of $1.55(\mu \mathrm{m})$ and power of $10 \mathrm{~mW}$ were released into the input port of the PANDA ring resonator circuit. The waveguide material used is $\operatorname{In} G a A s P / \operatorname{InP}$, with core index is $n_{0}=3.14$, core area of waveguide is $\mathrm{A}_{\text {eff }}=0.3$ $\mu \mathrm{m}^{2}$ and waveguide loss coefficient is $\alpha=0.1 \mathrm{dBmm}^{-1}$. The coupling coefficient ratios were $\kappa_{0}=0.018, \kappa_{1}=$ $0.44, \kappa_{2}=0.92, \kappa_{3}=0.39$.

In this simulation work, in order to provide a range of random radii consistent with the nature of the degrees of the sliding filament mechanism, we define the first ring is arranged as a reference ring (reference unit), with radius $R_{\mathrm{L}}=3.2499 \mu \mathrm{m}$. The second ring $R_{\mathrm{R}}$ is the sensing ring (sensing unit), which was position as the ring perturbed by the applied force (the muscle contraction). The ring radii varied from $3.2499 \mu \mathrm{m}$ to $3.2540 \mu \mathrm{m}$ and the third ring is used to form the interference signals between signals from the first ring and the second ring, with the radius $R_{\mathrm{ad}}=12 \mu \mathrm{m}$. Therefore, an "Affecter" degree of muscle perturbation is the effect caused by muscle contraction in the relaxation state's of the degrees of sliding filament mechanism. This perturbation has a random range of radii between $3.2499 \mu \mathrm{m}$ and $3.2522 \mu \mathrm{m}$. The "Minor" and the "Major" of muscle perturbation are the effects caused by muscle contraction when contracting to the fully contracted state. These perturbations have a 
Table 4 The relationship between the degrees of sensor impact and the random range of radius

\begin{tabular}{ccc}
\hline $\begin{array}{c}\text { Degree of sensor } \\
\text { perturbation }\end{array}$ & $\begin{array}{c}\text { The percentage of the } \\
\text { sarcomere length }\end{array}$ & Random range of radii \\
\hline Affecter & 58.82 & $3.2499-3.2522$ \\
Minor & 24.37 & $3.2523-3.2533$ \\
Major & 16.81 & $3.2534-3.2540$ \\
\hline
\end{tabular}

random range of radii between $3.2523 \mu \mathrm{m}$ and 3.2533 $\mu \mathrm{m}$ for the "Minor" state and $3.2534 \mu \mathrm{m}$ to $2540 \mu \mathrm{m}$ for the "Major" state. This is shown in Column 3 of Table 4.

In Table 4, the random range of radii is obtained from the relationship between the degrees of the perturbation, which occurred on the sensing unit $\left(E_{\mathrm{R}}\right)$ within the optical muscle sensing system and the percentage of the sarcomere length. So, while the eight facial gestures (listed in Table 3 ) was conducted the signals of the facial gesture is collected from all probes obtained the different degree of sensor perturbation is placed in various positions on the face. In terms of measurement methods, these received different signals are based on the difference of the facial gestures. In order to make the facial gesture signal values for the simulation as accurate as the signal values collected from the measurements of the actual situation, we have randomized the range of the second ring (sensing unit) radius based on the degree of sensor perturbation from 1,500 occurrences of each facial gesture, as shown in Fig. 7. In addition, individual physical differences will directly affect the movement of the facial gesture in real situations. We have demonstrated comparing the percentage of varied overlap. This relationship is shown in Table 5. Typical simulation results of overlap radii generated are shown in Fig. 8-11.

\section{Data Interpretation and Discussion}

From the previous section, the simulation results of the facial gesture demonstrate the differences from a wide variety of data, both in terms of the amount of data and the volume of the parameter, as shown in Fig. 7-11. In Fig. 7 shows an example of signal data pattern detected by the 11 probes, which have a different radius based on the degrees of perturbation. The "closing both eyes" gestures effect a "Major" degrees in probes "P3", "P4", and "P5" "Minor" degrees in probes "P1", "P2", "P6", "P7", "P8", and "P9" and other probes are "Affecter" degrees, which is the radius of the sensing ring is random in the range of $3.2534 \mu \mathrm{m}-3.2540 \mu \mathrm{m}, 3.2523 \mu \mathrm{m}-3.2533$ $\mu \mathrm{m}$ and $3.2499 \mu \mathrm{m}-3.2522 \mu \mathrm{m}$, respectively. The results of the data that has been created in the first record of the "closing both eyes" gesture to have the

\begin{tabular}{|r|c|c|c|c|c|c|c|c|c|c|c|}
\hline & P1 & P2 & P3 & P4 & P5 & P6 & P7 & P8 & P9 & P10 & P11 \\
\hline 1 & 3.2533 & 3.2524 & 3.2537 & 3.2538 & 3.2534 & 3.2525 & 3.2529 & 3.2531 & 3.2532 & 3.2511 & 3.2511 \\
\hline 2 & 3.2529 & 3.2523 & 3.2536 & 3.2537 & 3.2535 & 3.2533 & 3.2526 & 3.2529 & 3.2529 & 3.2505 & 3.2516 \\
\hline 3 & 3.2533 & 3.2526 & 3.2539 & 3.2536 & 3.2537 & 3.2528 & 3.2528 & 3.2530 & 3.2531 & 3.2504 & 3.2417 \\
\hline 4 & 3.2529 & 3.2524 & 3.2539 & 3.2539 & 3.2540 & 3.2527 & 3.2527 & 3.2529 & 3.2524 & 3.2505 & 3.2508 \\
\hline 5 & 3.2525 & 3.2531 & 3.2539 & 3.2539 & 3.2536 & 3.2532 & 3.2526 & 3.2530 & 3.2528 & 3.2515 & 3.2510 \\
\hline 6 & 3.2524 & 3.2531 & 3.2536 & 3.2538 & 3.2536 & 3.2524 & 3.2533 & 3.2527 & 3.2523 & 3.2509 & 3.2519 \\
\hline 7 & 3.2524 & 3.2526 & 3.2534 & 3.2539 & 3.2535 & 3.2531 & 3.2528 & 3.2533 & 3.2525 & 3.2517 & 3.2504 \\
\hline 8 & 3.2531 & 3.2526 & 3.2538 & 3.2536 & 3.2538 & 3.2525 & 3.2524 & 3.2532 & 3.2532 & 3.2520 & 3.2500 \\
\hline & $\vdots$ & $\vdots$ & $\vdots$ & $\vdots$ & $\vdots$ & $\vdots$ & $\vdots$ & $\vdots$ & $\vdots$ & $\vdots$ & $\vdots$ \\
\hline 1496 & 3.2523 & 3.2526 & 3.2539 & 3.2537 & 3.2535 & 3.2527 & 3.2533 & 3.2533 & 3.2527 & 3.2518 & 3.2515 \\
\hline 1497 & 3.2532 & 3.2526 & 3.2538 & 3.2539 & 3.2534 & 3.2526 & 3.2530 & 3.2531 & 3.2526 & 3.2509 & 3.2520 \\
\hline 1498 & 3.2525 & 3.2525 & 3.2536 & 3.2539 & 3.2539 & 3.2533 & 3.2532 & 3.2530 & 3.2530 & 3.2517 & 3.2513 \\
\hline 1499 & 3.2528 & 3.2527 & 3.2538 & 3.2535 & 3.2537 & 3.2532 & 3.2533 & 3.2529 & 3.2532 & 3.2515 & 3.2513 \\
\hline 1500 & 3.2529 & 3.2532 & 3.2535 & 3.2534 & 3.2534 & 3.2529 & 3.2524 & 3.2527 & 3.2529 & 3.2520 & 3.2505 \\
\hline
\end{tabular}

Fig. 7 Non-overlapping in "closing both eyes" gestures.

Table 5 The random range of radius based on the percentage of overlap radius

\begin{tabular}{ccccc}
\hline \multirow{2}{*}{ Degree of perturbation } & Random range of radii & \multicolumn{2}{c}{ The percentage of overlap radii } \\
\cline { 3 - 5 } & & $20 \%$ & $30 \%$ & $40 \%$ \\
\hline Affecter & $3.2499-3.2522$ & $3.2499-3.2524$ & $3.2499-3.2525$ & $3.2499-3.2526$ \\
Minor & $3.2523-3.2533$ & $3.2518-3.2534$ & $3.2516-3.2535$ & $3.2513-3.2536$ \\
Major & $3.2534-3.2540$ & $3.2532-3.2540$ & $3.2531-3.2540$ & $3.2530-3.2540$ \\
\hline
\end{tabular}




\begin{tabular}{|r|c|c|c|c|c|c|c|c|c|c|c|}
\hline & P1 & P2 & P3 & P4 & P5 & P6 & P7 & P8 & P9 & P10 & P11 \\
\hline 1 & 3.2533 & 3.2521 & 3.2537 & 3.2536 & 3.2538 & 3.2532 & 3.2519 & 3.2522 & 3.2526 & 3.2521 & 3.2508 \\
\hline 2 & 3.2528 & 3.2524 & 3.2535 & 3.2536 & 3.2533 & 3.2523 & 3.2533 & 3.2525 & 3.2532 & 3.2509 & 3.2524 \\
\hline 3 & 3.2527 & 3.2526 & 3.2538 & 3.2534 & 3.2535 & 3.2527 & 3.2525 & 3.2527 & 3.2519 & 3.2503 & 3.2499 \\
\hline 4 & 3.2521 & 3.2522 & 3.2532 & 3.2535 & 3.2537 & 3.2528 & 3.2527 & 3.2518 & 3.2529 & 3.2521 & 3.2511 \\
\hline 5 & 3.2526 & 3.2530 & 3.2538 & 3.2533 & 3.2537 & 3.2519 & 3.2521 & 3.2522 & 3.2532 & 3.2505 & 3.2505 \\
\hline 6 & 3.2534 & 3.2531 & 3.2538 & 3.2536 & 3.2535 & 3.2534 & 3.2519 & 3.2522 & 3.2523 & 3.2512 & 3.2512 \\
\hline 7 & 3.2526 & 3.2522 & 3.2535 & 3.2540 & 3.2539 & 3.2530 & 3.2524 & 3.2529 & 3.2520 & 3.2500 & 3.2502 \\
\hline 8 & 3.2523 & 3.2531 & 3.2533 & 3.2533 & 3.2539 & 3.2533 & 3.2525 & 3.2521 & 3.2522 & 3.2504 & 3.2523 \\
\hline & $\vdots$ & $\vdots$ & $\vdots$ & $\vdots$ & $\vdots$ & $\vdots$ & $\vdots$ & $\vdots$ & $\vdots$ & $\vdots$ & $\vdots$ \\
\hline 1496 & 3.2531 & 3.2531 & 3.2539 & 3.2539 & 3.2533 & 3.2526 & 3.2525 & 3.2534 & 3.2531 & 3.2503 & 3.2504 \\
\hline 1497 & 3.2519 & 3.2521 & 3.2539 & 3.2532 & 3.2540 & 3.2531 & 3.2532 & 3.2532 & 3.2524 & 3.2510 & 3.2508 \\
\hline 1498 & 3.2519 & 3.2526 & 3.2539 & 3.2533 & 3.2539 & 3.2531 & 3.2523 & 3.2522 & 3.2522 & 3.2513 & 3.2519 \\
\hline 1499 & 3.2524 & 3.2524 & 3.2540 & 3.2532 & 3.2538 & 3.2532 & 3.2530 & 3.2526 & 3.2529 & 3.2502 & 3.2520 \\
\hline 1500 & 3.2529 & 3.2527 & 3.2533 & 3.2536 & 3.2534 & 3.2520 & 3.2526 & 3.2531 & 3.2520 & 3.2520 & 3.2518 \\
\hline
\end{tabular}

Fig. 8 Overlapping $20 \%$ in "closing both eyes" gestures.

\begin{tabular}{|r|c|c|c|c|c|c|c|c|c|c|c|}
\hline & P1 & P2 & P3 & P4 & P5 & P6 & P7 & P8 & P9 & P10 & P11 \\
\hline 1 & 3.2530 & 3.2518 & 3.2536 & 3.2534 & 3.2538 & 3.2532 & 3.2518 & 3.2523 & 3.2530 & 3.2520 & 3.2509 \\
\hline 2 & 3.2525 & 3.2530 & 3.2536 & 3.2537 & 3.2539 & 3.2518 & 3.2530 & 3.2524 & 3.2522 & 3.2506 & 3.2499 \\
\hline 3 & 3.2523 & 3.2530 & 3.2535 & 3.2534 & 3.2533 & 3.2524 & 3.2518 & 3.2527 & 3.2520 & 3.2504 & 3.2513 \\
\hline 4 & 3.2527 & 3.2516 & 3.2534 & 3.2532 & 3.2538 & 3.2520 & 3.2531 & 3.2531 & 3.2523 & 3.2525 & 3.2523 \\
\hline 5 & 3.2516 & 3.2534 & 3.2539 & 3.2538 & 3.2532 & 3.2531 & 3.2529 & 3.2521 & 3.2535 & 3.2516 & 3.2508 \\
\hline 6 & 3.2520 & 3.2528 & 3.2540 & 3.2540 & 3.2533 & 3.2530 & 3.2521 & 3.2530 & 3.2520 & 3.2500 & 3.2507 \\
\hline 7 & 3.2534 & 3.2522 & 3.2534 & 3.2537 & 3.2531 & 3.2523 & 3.2522 & 3.2527 & 3.2529 & 3.2505 & 3.2510 \\
\hline 8 & 3.2532 & 3.2534 & 3.2536 & 3.2535 & 3.2540 & 3.2524 & 3.2531 & 3.2527 & 3.2533 & 3.2517 & 3.2503 \\
\hline & $\vdots$ & $\vdots$ & $\vdots$ & $\vdots$ & $\vdots$ & $\vdots$ & $\vdots$ & $\vdots$ & $\vdots$ & $\vdots$ & $\vdots$ \\
\hline 1496 & 3.2527 & 3.2524 & 3.2533 & 3.2534 & 3.2536 & 3.2529 & 3.2523 & 3.2521 & 3.2525 & 3.2502 & 3.2521 \\
\hline 1497 & 3.2531 & 3.2517 & 3.2535 & 3.2531 & 3.2538 & 3.2530 & 3.2528 & 3.2529 & 3.2521 & 3.2517 & 3.2518 \\
\hline 1498 & 3.2523 & 3.2519 & 3.2535 & 3.2539 & 3.2539 & 3.2519 & 3.2517 & 3.2517 & 3.2529 & 3.2508 & 3.2525 \\
\hline 1499 & 3.2525 & 3.2522 & 3.2534 & 3.2538 & 3.2539 & 3.2532 & 3.2535 & 3.2528 & 3.2528 & 3.2514 & 3.2502 \\
\hline 1500 & 3.2518 & 3.2519 & 3.2538 & 3.2536 & 3.2537 & 3.2534 & 3.2519 & 3.2528 & 3.2532 & 3.2514 & 3.2518 \\
\hline
\end{tabular}

Fig. 9 Overlapping 30\% in "closing both eyes" gestures.

\begin{tabular}{|r|c|c|c|c|c|c|c|c|c|c|c|}
\hline & P1 & P2 & P3 & P4 & P5 & P6 & P7 & P8 & P9 & P10 & P11 \\
\hline 1 & 3.2515 & 3.2516 & 3.2538 & 3.2535 & 3.2535 & 3.2528 & 3.2521 & 3.2514 & 3.2520 & 3.2517 & 3.2514 \\
\hline 2 & 3.2521 & 3.2535 & 3.2537 & 3.2536 & 3.2533 & 3.2519 & 3.2518 & 3.2532 & 3.2535 & 3.2516 & 3.2507 \\
\hline 3 & 3.2520 & 3.2533 & 3.2540 & 3.2532 & 3.2537 & 3.2523 & 3.2529 & 3.2527 & 3.2535 & 3.2512 & 3.2523 \\
\hline 4 & 3.2533 & 3.2531 & 3.2532 & 3.2536 & 3.2534 & 3.2528 & 3.2523 & 3.2515 & 3.2516 & 3.2510 & 3.2512 \\
\hline 5 & 3.2533 & 3.2528 & 3.2538 & 3.2538 & 3.2532 & 3.2514 & 3.2519 & 3.2534 & 3.2514 & 3.2526 & 3.2515 \\
\hline 6 & 3.2529 & 3.2523 & 3.2534 & 3.2534 & 3.2536 & 3.2525 & 3.2520 & 3.2535 & 3.2533 & 3.2508 & 3.2523 \\
\hline 7 & 3.2520 & 3.2522 & 3.2538 & 3.2532 & 3.2533 & 3.2517 & 3.2529 & 3.2515 & 3.2533 & 3.2515 & 3.2521 \\
\hline 8 & 3.2528 & 3.2514 & 3.2532 & 3.2531 & 3.2537 & 3.2529 & 3.2534 & 3.2531 & 3.2535 & 3.2500 & 3.2512 \\
\hline & $\vdots$ & $\vdots$ & $\vdots$ & $\vdots$ & $\vdots$ & $\vdots$ & $\vdots$ & $\vdots$ & $\vdots$ & $\vdots$ & $\vdots$ \\
\hline 1496 & 3.2535 & 3.2517 & 3.2531 & 3.2534 & 3.2535 & 3.2526 & 3.2535 & 3.2532 & 3.2516 & 3.2518 & 3.2508 \\
\hline 1497 & 3.2523 & 3.2522 & 3.2531 & 3.2531 & 3.2531 & 3.2526 & 3.2520 & 3.2534 & 3.2535 & 3.2505 & 3.2520 \\
\hline 1498 & 3.2528 & 3.2520 & 3.2535 & 3.2539 & 3.2535 & 3.2515 & 3.2527 & 3.2535 & 3.2531 & 3.2526 & 3.2522 \\
\hline 1499 & 3.2525 & 3.2515 & 3.2539 & 3.2532 & 3.2538 & 3.2529 & 3.2521 & 3.2530 & 3.2524 & 3.2504 & 3.2512 \\
\hline 1500 & 3.2518 & 3.2525 & 3.2535 & 3.2533 & 3.2535 & 3.2522 & 3.2536 & 3.2527 & 3.2519 & 3.2521 & 3.2518 \\
\hline
\end{tabular}

Fig. 10 Overlapping 40\% in "closing both eyes" gestures.

signal data pattern in each probe from "P1" to "P11" is "3.2533", “3.2524”, “3.2537”, "3.2538”, "3.2534", “3.2525”, “3.2529”, “3.2531”, “3.2532”, “3.2511”, and " 3.2511 ", respectively. In the second record of the same gesture, the data signal received is " 3.2529 ", “3.2523”, “3.2536”, “3.2537”, “3.2535”, “3.2533”, "3.2526", "3.2529", "3.2529”, "3.2505”, and "3.2516", respectively. So, the twelve thousand signal data patterns were obtained from 11 probes of the 8 facial gestures through the simulation of facial gestures 1500 times in one gesture. In addition, we also recognize the individual differences theory because these gesture would have to be different in the amount of force used in the process of muscle contraction. We assume that a situation of overlapping in degrees of perturbation has occurred, it has defined as the ratio of the overlap is $20 \%, 30 \%, 40 \%$, and $50 \%$, which results are shown in Fig. 8-11, respectively. The results of these simulations 


\begin{tabular}{|r|c|c|c|c|c|c|c|c|c|c|c|}
\hline & P1 & P2 & P3 & P4 & P5 & P6 & P7 & P8 & P9 & P10 & P11 \\
\hline 1 & 3.2523 & 3.2513 & 3.2539 & 3.2536 & 3.2538 & 3.2536 & 3.2530 & 3.2537 & 3.2526 & 3.2520 & 3.2512 \\
\hline 2 & 3.2515 & 3.2521 & 3.2534 & 3.2530 & 3.2530 & 3.2515 & 3.2525 & 3.2511 & 3.2533 & 3.2516 & 3.2501 \\
\hline 3 & 3.2522 & 3.2535 & 3.2536 & 3.2539 & 3.2528 & 3.2524 & 3.2511 & 3.2532 & 3.2511 & 3.2499 & 3.2526 \\
\hline 4 & 3.2524 & 3.2528 & 3.2536 & 3.2532 & 3.2530 & 3.2528 & 3.2524 & 3.2530 & 3.2534 & 3.2522 & 3.2522 \\
\hline 5 & 3.2525 & 3.2530 & 3.2538 & 3.2538 & 3.2539 & 3.2526 & 3.2535 & 3.2535 & 3.2517 & 3.2526 & 3.2509 \\
\hline 6 & 3.2535 & 3.2512 & 3.2540 & 3.2533 & 3.2528 & 3.2528 & 3.2518 & 3.2519 & 3.2530 & 3.2519 & 3.2511 \\
\hline 7 & 3.2535 & 3.2518 & 3.2538 & 3.2534 & 3.2531 & 3.2534 & 3.2530 & 3.2526 & 3.2528 & 3.2527 & 3.2524 \\
\hline 8 & 3.2531 & 3.2529 & 3.2540 & 3.2535 & 3.2539 & 3.2514 & 3.2536 & 3.2524 & 3.2526 & 3.2511 & 3.2507 \\
\hline & $\vdots$ & $\vdots$ & $\vdots$ & $\vdots$ & $\vdots$ & $\vdots$ & $\vdots$ & $\vdots$ & $\vdots$ & $\vdots$ & $\vdots$ \\
\hline 1496 & 3.2534 & 3.2536 & 3.2531 & 3.2534 & 3.2530 & 3.2515 & 3.2536 & 3.2532 & 3.2515 & 3.2513 & 3.2514 \\
\hline 1497 & 3.2535 & 3.2515 & 3.2530 & 3.2535 & 3.2533 & 3.2519 & 3.2522 & 3.2526 & 3.2516 & 3.2524 & 3.2513 \\
\hline 1498 & 3.2520 & 3.2529 & 3.2530 & 3.2537 & 3.2534 & 3.2524 & 3.2532 & 3.2529 & 3.2512 & 3.2526 & 3.2518 \\
\hline 1499 & 3.2529 & 3.2523 & 3.2531 & 3.2538 & 3.2539 & 3.2529 & 3.2518 & 3.2521 & 3.2520 & 3.2515 & 3.2503 \\
\hline 1500 & 3.2534 & 3.2534 & 3.2536 & 3.2533 & 3.2538 & 3.2534 & 3.2530 & 3.2528 & 3.2535 & 3.2528 & 3.2504 \\
\hline
\end{tabular}

Fig. 11 Overlapping 50\% in "closing both eyes" gestures.

have shown clearly that there is a random distribution of various radii of the ring and to cover the possibility of the degrees of perturbation in a real situation. This amount of data and volume of parameters caused difficulties in the processing and interpretation of the data. However, the various data problems can be handled effectively by data mining techniques (DMT). The classification function is one of the outstanding techniques of data mining, which is used to classify data into pre-identifiable categorical class labels.

In application of data mining techniques, the extent and variety of the facial gesture signals resulting from the simulations show that these data mining methods can be used in the analysis of this data, assisting in the improvement and development of processes of the assistive technology or tools in order to have the ability of the signal pattern recognition based on the machine learning approaches. This is important data to be applied to research and resolve issues with various assistance systems such as human-robot interaction (HRI), human computer interaction (HCI), and human machine interaction (HMI) in the near future.

\section{Conclusions}

The overall objective of our work is to demonstrate an interesting approach of facial gesture measurement, which can be a good candidate for optical sensing system. In principle, the optical muscle sensing system was performed based on the change in wavelength of the optical sensor can be configured to the optical path length variation within the PANDA ring resonator. The results obtained from the simulation of the facial gesture measurement are performed based on the actual situation of the mechanism of the muscle contraction. The relationship between the shortening of the sarcomere and the sarcomere length was applied as the basis concept to determine the perturbed degrees of the muscle contraction, which is divided into three levels, namely affecter, minor, and major. The process of sampling to adjust the size of the radius within the sensing unit will be used to demonstrate the changes that occur within the sensing system after perturbed by the muscle contraction. In future work, we intend to apply these signal data were obtained from the simulation with the machine learning approaches in order to classify and recognize the facial gesture signal pattern, which will be extremely useful for various utilization such as hands-free human-computer interactions and behavior monitoring which can be used in many disability support applications.

\section{Acknowledgements}

This work is partially supported by the Faculty of Science, Naresuan University, Thailand. Lastly, the authors also gratefully acknowledge the Faculty of Science, King Mongkut's Institute of Technology Ladkrabang, Thailand, for the laboratory and research facilities. Many thanks to Mr. Roy Irvine Morien of the Naresuan University Language Centre for his editing assistance and advice on English expression in this document.

\section{References}

[1] S. Berman, H. Stern, Sensors for Gesture Recognition Systems. IEEE Transactions on Systems, Man, and Cybernetics, Part C: Applications and Reviews, 2012, 42(3): 277-290.

[2] C. Cipriani, M. D’Alonzo, M. C. Carrozza, A Miniature Vibrotactile Sensory Substitution Device for Multifingered 
Hand Prosthetics. IEEE Transactions on Biomedical Engineering, 2012, 59(2): 400-408.

[3] B. Hafidh, H. Al Osman, M. Alowaidi, et al., F-Glove: A glove with force-audio sensory substitution system for diabetic patients. In 2013 IEEE International Symposium on Haptic Audio Visual Environments and Games (HAVE), 2013.

[4] H. Jabnoun, F. Benzarti, H. Amiri, Visual substitution system for blind people based on SIFT description. In 2014 6th International Conference of Soft Computing and Pattern Recognition (SoCPaR), 2014.

[5] E. Causevic, R. E. Morley, M. V. Wickerhauser, et al., Fast wavelet estimation of weak biosignals. IEEE Transactions on Biomedical Engineering, 2005, 52(6): 1021-1032.

[6] F. N. Jamaluddin, S. A. Ahmad, S. B. M. Noor, et al., Flexible bio-signals channels acquisition system for ECG and EMG application. In 2014 IEEE Student Conference on Research and Development (SCOReD), 2014.

[7] M. Kutz, BIOMEDICAL SIGNAL ANALYSIS. In Standard Handbook of Biomedical Engineering \& Design, McGraw Hill Professional, Access Engineering, 2003.

[8] S. U. Kumar, V. M. Vinod, EOG based wheelchair control for quadriplegics. In 2015 International Conference on Innovations in Information, Embedded and Communication Systems (ICIIECS), 2015.

[9] A. Marjaninejad, S. Daneshvar, A low-cost real-time wheelchair navigation system using electrooculography. In 2014 22nd Iranian Conference on Electrical Engineering (ICEE), 2014.

[10] B. Hafidh, H. Al Osman, M. Alowaidi, et al., F-Glove: A glove with force-audio sensory substitution system for diabetic patients. In 2013 IEEE International Symposium on Haptic Audio Visual Environments and Games (HAVE), 2013.

[11] H. Jabnoun, F. Benzarti, H. Amiri, Visual substitution system for blind people based on SIFT description. In 2014 6th International Conference of Soft Computing and Pattern Recognition (SoCPaR), 2014.

[12] L. Twardon, H. Koesling, A. Finke, et al., Gazecontingent audio-visual substitution for the blind and visually impaired. In 2013 7th International Conference on Pervasive Computing Technologies for Healthcare (PervasiveHealth), 2013.

[13] J. Lin, X. Ruan, N. Yu, et al., One-shot learning gesture recognition based on improved 3D SMoSIFT feature descriptor from RGB-D videos. In Control and Decision Conference (CCDC), 2015 27th Chinese, 2015.

[14] J.G. Bueno, M. Gonzalez-Fierro, C. Balaguer, et al., Facial gesture recognition using active appearance models based on neural evolution. In 20127 th ACM/IEEE International Conference on Human-Robot Interaction (HRI), 2012.

[15] Y.B. Lee, S.B. Moon, Y.G. Kim, Face and Facial Expression Recognition with an Embedded system for Human-Robot Interaction. In Affective Computing and Intelligent Interaction, J. Tao, T. Tan, and R. W. Picard, Eds. Springer Berlin Heidelberg, 2005.

[16] M. Goel, C. Zhao, R. Vinisha, et al., Tongue-in-Cheek: Using Wireless Signals to Enable Non-Intrusive and Flexible Facial Gestures Detection. In Proceedings of the 33rd Annual ACM Conference on Human Factors in Computing Systems, New York, NY, USA, 2015.

[17] U. Agrawal, S. Giripunje, P. Bajaj, Emotion and Gesture Recognition with Soft Computing Tool for Drivers Assistance System in Human Centered Transportation. In 2013 IEEE International Conference on Systems, Man, and Cybernetics (SMC), 2013.

[18] V.C. Sendra, C. Kaland, M. Swerts, et al., Perceiving incredulity: The role of intonation and facial gestures. Journal of Pragmatics, 2013, 47(1): 1-13.

[19] J.G. Bueno, M.G. Fierro, C. Balaguer, et al., Facial gesture recognition using active appearance models based on neural evolution. In 2012 7th ACM/IEEE International Conference on Human-Robot Interaction (HRI), 2012.

[20] N. Sabri, S. A. Aljunid, M. S. Salim, et al., Toward Optical Sensors: Review and Applications. Journal of Physics: Conference Series (J. Phys.: Conf. Ser.), 2013, 423(1): 1-7.

[21] K. Srinuanjan, W. Tipaphong, S. Kamoldilok, et al., A self-calibration nano-scale sensing transducer using PANDA ring resonator. In 2010 International Conference on Enabling Science and Nanotechnology (ESciNano), 2010 .

[22] K. Tamee, K. Srinuanjan, S. Mitatha, et al., Distributed Sensors Using a PANDA Ring Resonator Type in Multiwavelength Router. IEEE Sensors Journal, 2011, 11(9): 1987-1992.

[23] S. Glomglome, Molecular distributed sensors using dark soliton array trapping tools. Journal of Biomedical Science and Engineering, 2011, 4(6): 472-478.

[24] K. Srinuanjan, S. Kamoldilok, W. Tipaphong, et al., A nano-scale transducer using a PANDA type ring resonator for gas sensor applications. Optik-International Journal for Light and Electron Optics, 2012, 123(6): 475-478.

[25] K. Kulrod, C. Sirawattananon, S. Mitatha, et al., Force Sensing Device Design using a Modified Add-Drop filter. Procedia Engineering, 2012, 32: 291-298.

[26] P. P. Yupapin, N. Sarapat, Novel micro-scale sensors using WGMS within the modified add-drop filter circuits. Microwave and Optical Technology Letters (Microw. Opt. Technol. Lett.), 2014, 56(1): 14-17.

[27] K. Tamee, K. Chaiwong, K. Yothapakdee, et al., Muscle Sensor Model Using Small Scale Optical Device for Pattern Recognitions. The Scientific World Journal, 2013, 2013(Oct.).

[28] K. Yothapakdee, P.P. Yupapin, K. Tamee, Muscle Sensing Device Design using a PANDA Ring Resonator System. In 2015 International Conference on Sensors and Mechanical Automation (ICSMA 2015), Thailand, 2015.

[29] H. E. Huxley, Electron microscope studies of the organisation of the filaments in striated muscle. Biochim. Biophys. Acta, 1953, 12(3): 387-394.

[30] A.F. Huxley, R. Niedergerke, Structural Changes in Muscle During Contraction: Interference Microscopy of Living Muscle Fibres. Nature, 1954, 173(4412): 971-973.

[31] A. F. Huxley, Muscle structure and theories of contraction. Prog Biophys Biophys Chem, 1957, 7: 255-318

[32] H. E. Huxley, The mechanism of muscular contraction. Science, 1969, 164(3886): 1356-1365.

[33] E. N. Marie, K. Hoehn, Human Anatomy and Physiology, 9th edition. San Francisco, Calif.: Pearson Education/ Benjamin Cummings, 2013.

[34] D. Junge, W.F.H.M. Mommaerts, U. Windhorst, Sensory Transduction and Neural Coding. In Comprehensive Human Physiology, P.D.R. Greger and P.D.U. Windhorst, Eds. Springer Berlin Heidelberg, 1996.

[35] D. Shier, J. Butler, R. Lewis, Hole's Human Anatomy and Physiology, Student Edition, 12th. Dubuque: Clencoe/ McGraw Hill, 2009.

Copyright(C) 2015 Kriengsak Yothapakdee, Preecha P. Yupapin and Kreangsak Tamee. This is an open-access article distributed under the terms of the Creative Commons Attribution License, which permits unrestricted use, distribution, and reproduction in any medium, provided the original author and source are credited. 